\title{
PLATAFORMA DE COMUNICAÇÃO SEM FIO APLICADA A SISTEMAS DE IRRIGAÇÃO
}

\author{
F. B. ROCHA ${ }^{1^{*}}$, R. S. SILVA ${ }^{1}$, A. M. AVELINO ${ }^{2}$ e C. M. COSTA ${ }^{2}$ \\ ${ }^{1}$ Universidade Federal do Rio grande do Norte - UFRN \\ ${ }^{2}$ Instituto Federal de Educação, Ciência e Tecnologia do Rio Grande do Norte - IFRN \\ felipebarbalho_12@hotmail.com*
}

Artigo submetido em fevereiro/2014 e aceito em outubro/2014

DOI: $10.15628 /$ holos.2014.1945

\section{RESUMO}

A utilização de novas tecnologias para projetos de automação é hoje uma tendência nos grandes centros urbanos. Porém, no campo, a agricultura carece do uso dessas tecnologias, inclusive, em sistemas e métodos de irrigação e de monitoramento de lavouras, dos quais são em sua grande maioria ultrapassados e inadequados. Consequentemente, isso afeta diretamente todo o setor econômico de uma sociedade. Com isso, faz-se necessário o desenvolvimento de um mecanismo automatizado de controle de irrigação, possibilitando uma maior eficiência e eficácia no processo de irrigação. Para tanto, o objetivo deste trabalho foi o desenvolvimento de um sistema de hardware e software capaz de tornar o processo de irrigação mais simples para o agricultor. Esse sistema consiste no controle e monitoramento da irrigação por meio de uma aplicação desenvolvida para computadores pessoais e através da internet, tendo em vista a mobilidade proporcionada para acesso do sistema em qualquer lugar do mundo. Além das vantagens práticas e econômicas, o sistema automatizado de irrigação possui um simples desenvolvimento, e utiliza materiais de baixo custo, o que torna viável sua implantação em grandes, médias e, inclusive, em pequenas lavouras.

PALAVRAS-CHAVE: Agricultura, Irrigação, Sistema Automatizado.

\section{WIRELESS COMMUNICATION PLATFORM APPLIED TO IRRIGATION SYSTEMS}

\begin{abstract}
The use of new technologies for automation is a trend in urban centers. However, in countryside, the agriculture lacks the use of these technologies, even in irrigation and field monitoring systems and methods, which are, mostly, outdated and inadequate. Consequently, this causes a negative impact on economic sector of whole a society. So it becomes necessary to develop an automated irrigation control, allowing greater efficiency and effectiveness in the whole irrigation process. For this purpose, the aim of this work was the development of a
\end{abstract}

system composed of hardware and software that can make the process simpler for the farmer. This system consists in the control and monitoring of irrigation through an application developed for personal computers and through the internet, considering the mobility provided to access the system from anywhere. In addition to the practical and economic advantages, the automated system has a simple development, and uses low cost materials, which makes feasible its implementation in large, medium or small areas farming.

KEYWORDS: Agriculture, Irrigation, Automated System. 


\section{INTRODUÇÃO}

Atualmente, as tecnologias de conexão sem fio vêm crescendo cada vez mais e abrangendo um maior contingente no setor econômico. Isso aliado ao crescente investimento em um mecanismo de controle universal, os dispositivos móveis, que agregam a cada versão, mais funções e facilidades para o usuário. Segundo pesquisa realizada pela CONECTA e pela WIN (Worldwide Independent Network of Market Research), plataforma do IBOPE, entre 2011 e 2012, no Brasil o crescimento no uso desses aparelhos saltou de $9 \%$ para $18 \%$.

Aliado ao uso de dispositivos móveis, as aplicações que alimentam esses aparelhos, estão cada vez mais localizados em datacenters, e através da internet o usuário tem acesso a esses recursos. Isso é efeito de uma tendência atual no mundo tecnológico: a Computação em Nuvem. Esse conceito apresenta inúmeras vantagens, entre elas, é o acesso de uma mesma aplicação com um mesmo banco de dados, por diferentes dispositivos, aumentando ainda mais o conceito de mobilidade.

Apesar das inúmeras facilidades da Computação em Nuvem, o desenvolvimento de software, seja ele para dispositivos móveis ou desktops, ainda tem sua devida importância, levando em conta que nem sempre os usuários poderão estar conectados à internet. Toda essa tecnologia nem sempre é aplicada a todos os setores econômicos, algumas áreas como a agricultura apresentam grande déficit tecnológico, principalmente para médios e pequenos produtores, pelos quais os meios utilizados para o controle e monitoramento de sistemas de irrigação são, em sua maioria, dotados de pouca tecnologia.

A criação de um sistema de irrigação automatizado para o controle de áreas agrícolas, não vem a ser uma nova descoberta, considerando a existência de um vasto mercado voltado para esta área, todavia por apresentar uma tecnologia de alto custo, torna-se de difícil acesso para pequenos e médios agricultores (SILVA et al., 2007).

Com isso, observa-se a necessidade de desenvolvimento de sistemas que agreguem novas tecnologias, buscando uma utilização mais eficiente dos recursos naturais, com um custo acessível para pequenos e médios agricultores e ao mesmo tempo compondo um sistema de irrigação automatizado mais eficiente.

Portanto faz-se necessária a criação de um sistema eficiente para controle e monitoramento de irrigação na agricultura, que possa atuar diretamente no meio e estabelecer conexão com o usuário, dando-lhe facilidade e praticidade que as novas tecnologias oferecem, por um baixo custo.

\section{ARQUITETURA E DESENVOVIMENTO DO SISTEMA DE IRRIGAÇÃO}

No desenvolvimento do sistema procurou-se atender as necessidades do agricultor, dandoIhe opções de controle e monitoramento de forma eficiente. Em tempo real o mesmo terá dados da sua lavoura/plantio e um mecanismo atuando de forma automática ou por meio de comandos em um software de computador a fim de controlar todo o processo de irrigação. 
O funcionamento do sistema se resume a leitura dos sensores no campo e as ações do usuário: de ativação e desativação da irrigação. A Figura 1 representa a arquitetura de funcionamento do sistema.

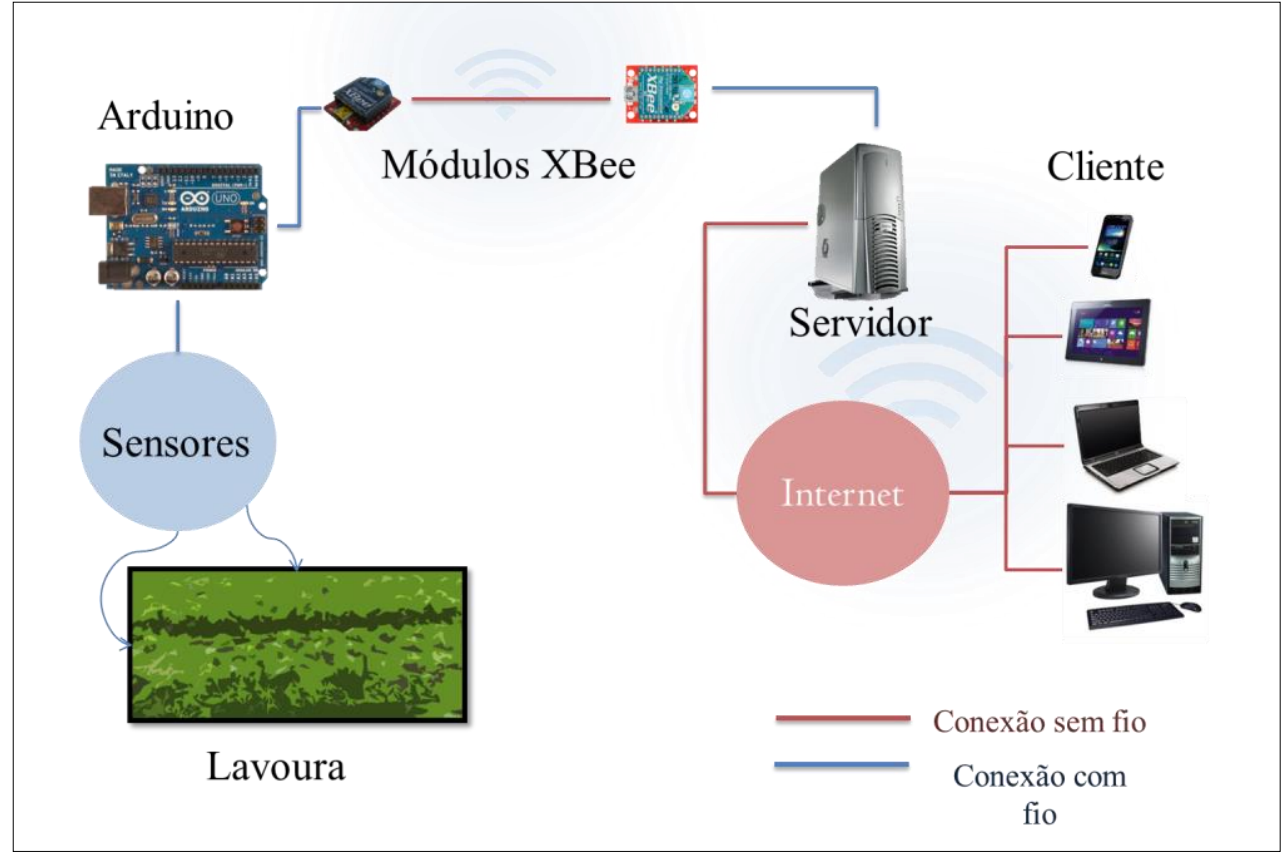

Figura 1 - Escopo de funcionamento do sistema.

Como se pode visualizar na figura acima, por meio de interfaces de comunicação sem fio, as informações cruzarão a extensão do campo até uma estação remota, que disponibilizará ao usuário toda uma iteratividade por aplicações e através da internet. Em tempo real o usuário terá controle da irrigação em sua lavoura.

Para tanto, o desenvolvimento e montagem do protótipo do Sistema de Irrigação se definiram em duas fases, Hardware e Software. Para desenvolvimento de cada fase foram utilizados mecanismos de Comunicação de Rede, Eletrônica digital e analógica, e Programação.

\section{HARDWARE}

Nessa fase foi feita uma seleção de material que atendesse a requisitos como preço, eficiência e facilidade de manejo por meio dos desenvolvedores. Os materiais escolhidos estão listados a seguir:

- Sensor de Umidade Honeywell HIH-4000;

- Sensor de Luminosidade LDR;

- Sensor de Temperatura, Termistor NTC 10K 2mm;

- Placa Arduino Uno;

- Módulo RTC DS1302;

- Módulos XBee.

\subsection{Sensoriamento}

No sistema elétrico, cada sensor é responsável pela leitura das variáveis de temperatura, luminosidade e umidade do local da automação, ou seja, do campo. A informação dos sensores é 
lida e interpretada por um microcontrolador, na placa Arduino UNO. A próxima subseção apresenta as principais características do Arduino UNO.

\subsection{Arduino UNO}

O funcionamento da fase de hardware desse sistema estará centralizado no processamento de dados pelo Arduino Uno. Mas por qual motivo esse sistema se torna viável através dessa placa para um projeto de controle e monitoramento de irrigação, tendo em vista a grande quantidade de sistemas microcontroladores existentes no mercado?

Segundo (LIMA et al., 2012), o Arduino é uma plataforma de prototipação eletrônica de código aberto (open-source), qualquer pessoa pode produzi-la, é fácil de programar e usar, além de possuir preço acessível e ser facilmente encontrada.

O Arduino UNO tem como mecanismo, um microcontrolador ATMEGA, desenvolvido pela Atmel Corporation, que permite ao desenvolvedor criar diversos tipos sistemas automatizado, robôs e etc. Ele possui uma porta de comunicação USB para se conectar a um computador, por um cabo USB-AB, e o desenvolvedor pode programar a função do microcontrolador na linguagem de programação própria do Arduino. Este último pode ser visualizado na Figura 2.

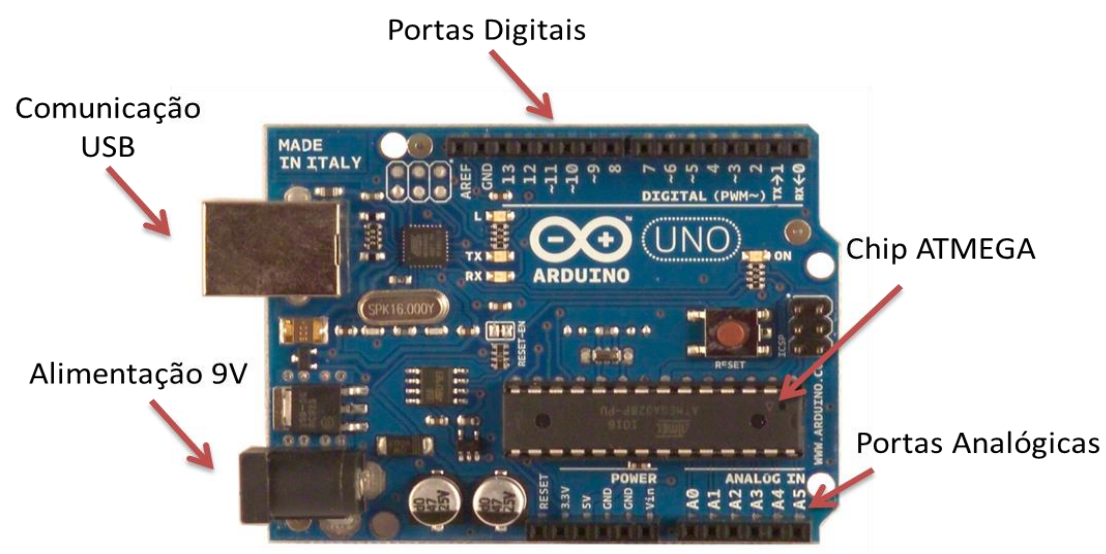

Figura 2: Arduino UNO.

O Arduino irá ler os valores dos sensores pelas portas analógicas, conforme ilustrado na figura anterior, e converte-los (utilizando o conversor analógico-digital interno) em um valor digital compreendido entre 0 (correspondente a OV) e 1023 (correspondente a 5V). O código embarcado no Arduino fará um tratamento desses valores, esse processo será descrito mais à frente na seção de software.

Os dados serão enviados por meio de comunicação serial a cada três segundos, para comunicação com o software do servidor. Para essa tarefa será utilizada uma interface de comunicação sem fio. Para tal será utilizado Módulos XBee.

\subsection{Módulos XBee}

Esses módulos utilizam o padrão ZigBee, desenvolvido para se tornar uma alternativa de comunicação em redes que não necessitem de soluções mais complexas para seu controle, barateando assim os custos com a aquisição, instalação de equipamentos, manutenção e mão de obra. Trata-se de uma tecnologia relativamente simples, que utiliza um protocolo de pacotes de 
dados com características específicas, sendo projetado para oferecer flexibilidade quanto aos tipos de dispositivos que pode controlar. Os dispositivos baseados na tecnologia ZigBee operam na faixa ISM (Industrial, Scientific and Medical) que não requer licença para funcionamento, incluindo as faixas de $2,4 \mathrm{GHz}$ (Global), $915 \mathrm{MHz}$ (América) e $868 \mathrm{MHz}$ (Europa) e com taxas de transferência de dados de 250Kbps em 2,4GHz (PINHEIRO, 2004).

O módulo XBee funciona como uma antena de comunicação para longas distâncias, ele substitui o cabo USB, que serve como a camada física de comunicação serial entre o servidor e o Arduino. Um XBee com potência de $1 \mathrm{~mW}$ proporciona uma área de cobertura de $100 \mathrm{~m}$, considerando um meio físico sem obstruções. A comunicação se dá entre dois ou mais módulos XBee. O mesmo pode ser visualizado na Figura 3.

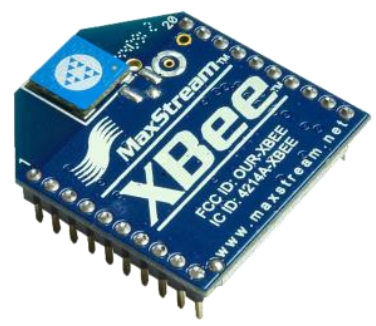

Figura 3. XBee.

Um módulo XBee será plugado junto com a Shield - XBee SF (placa auxiliar que permite ligar - XBee ao Arduino). Outro módulo será plugado no XBee Explorer USB (placa auxiliar que permite ligar o XBee ao servidor), e ficará conectado a uma porta USB. O esquema de conexão pode ser visualizado na Figura 4.

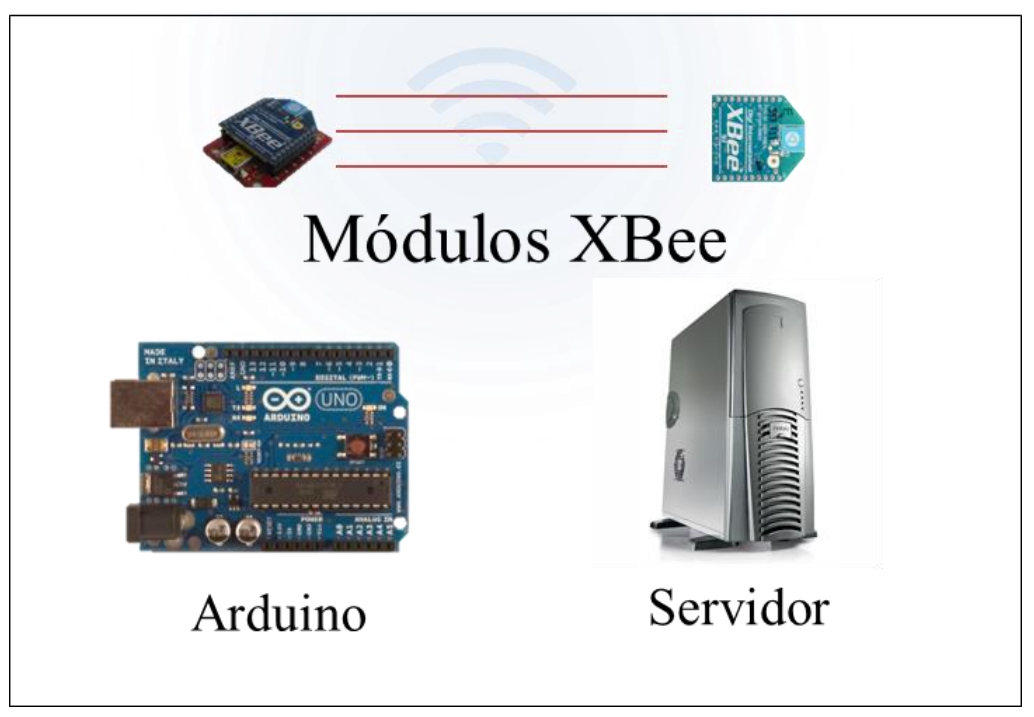

Figura 4: Comunicação ZigBee.

\subsection{Comunicação Serial}

A comunicação entre o XBee Explorer USB e o computador será feita através da comunicação RS232. O módulo XBee Explorer USB é reconhecido pelo computador como uma porta serial (RS232). A comunicação implementada é half-duplex, e segundo (Lista, 2007), esse fato limita a transmissão de grandes quantidades de informações em pequenos intervalos de tempo, 
devido a isso é fundamental a utilização de uma estrutura na transmissão simples, para evitar possíveis falhas, pela sobrecarga na transmissão dos dados. Quanto à segurança será utilizado a encriptação AES 128-bits entre os módulos.

\subsection{Módulo RTC DS1302}

O módulo RTC DS1302 é um importante componente responsável pela automatização da irrigação, nele atua o CI DS1307, com a finalidade de contabilizar o tempo, incluindo data e dia da semana, uma bateria de lítio tipo moeda mantém o mesmo funcionando por anos. Uma llustração do Módulo RTC (Real Time Clock) pode ser visualizada na Figura 5.

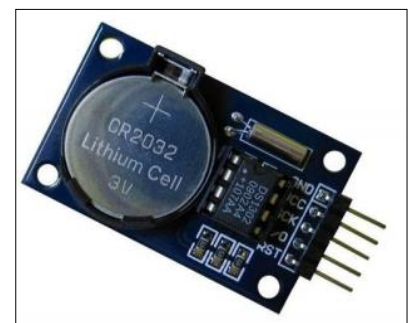

Figura 5 - Módulo RTC DS1302.

Utilizando o RTC é possível assegurar que a contagem de tempo continue mesmo com o desligamento da alimentação do Arduino (em caso de falta de energia, por exemplo), já que o módulo possui uma bateria de backup.

Com isso foi possível estabelecer um calendário de irrigação, em que os horários de ativação e desativação fossem previamente configurados, e executados no horário estabelecido. Dessa forma, o esquema final da montagem do protótipo pode ser visualizado como apresentado na Figura 6.

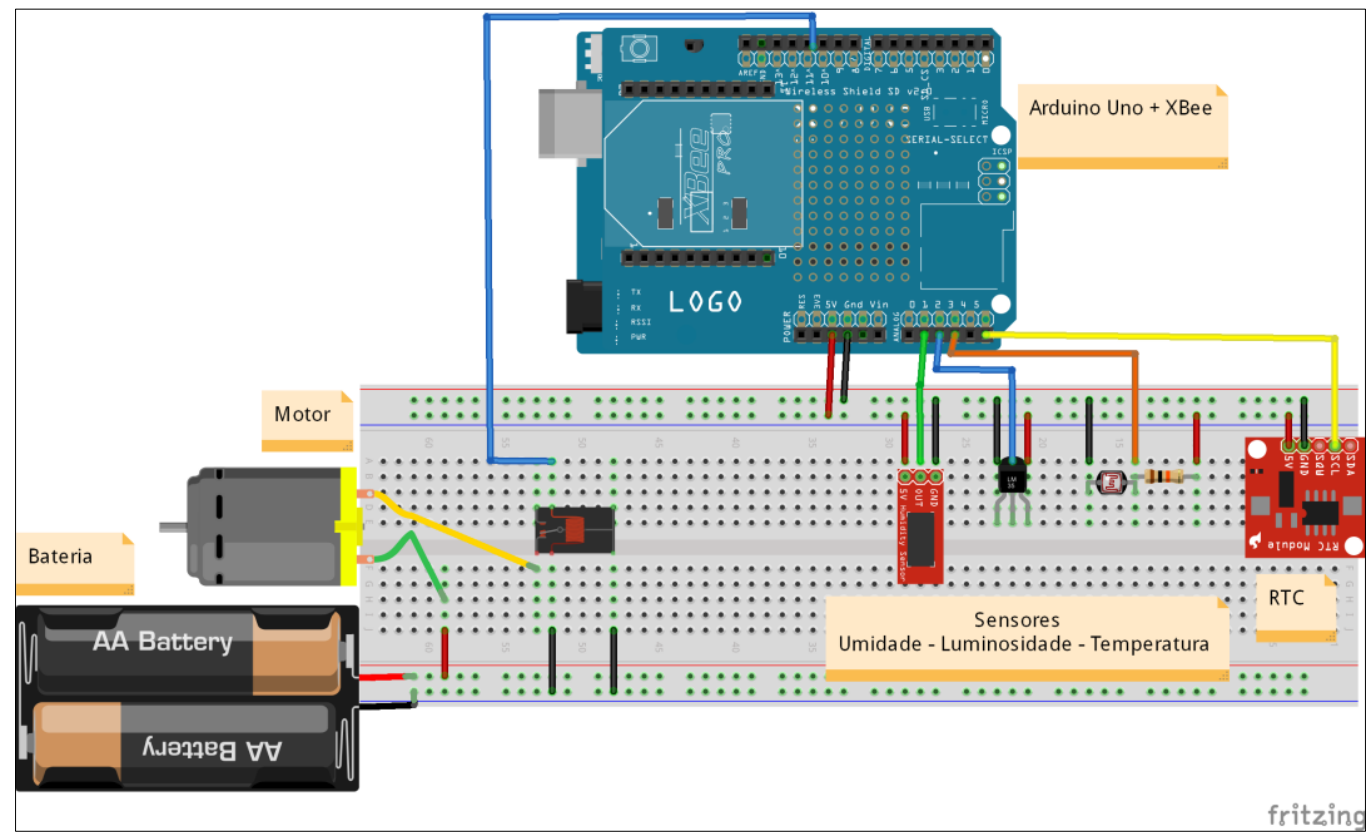

Figura 6 - Esquema de Prototipagem do sistema de Hardware.

No esquema anterior além dos componentes que foram citados, há presença de um motor DC (Direct Current), representando uma bomba comumente utilizada na irrigação, baterias para 
representação da alimentação independente do Arduino. Um relé funciona como um interruptor, que ao receber um pulso de $5 \mathrm{~V}$ da porta digital do Arduino, libera a corrente proveniente da bateria, acionando o motor. Por meio do relé é possível acionar circuitos com tensões de até a $250 \mathrm{~V}$ e correntes até $10 \mathrm{~A}$.

\subsection{Firmware}

A irrigação no modo automático é um meio independente da comunicação com o usuário, dessa forma ela precisa do maior número possível de informações e de controle de falhas para proporcionar um sistema robusto, e econômico quanto aos recursos hídricos e de energia elétrica.

A economia de agua no processo de irrigação não obedece a uma forma padrão, ela depende da cultura, do método de irrigação, do nível de radiação de luz solar, e da confiabilidade do sistema de controle. Porém é importante salientar, que a gestão da agua no sistema de irrigação não pode interferir na qualidade do produto cultivado, ela deve somente evitar eventuais desperdícios de agua.

Para tal, o sistema de controle necessita de alguns parâmetros previamente configurados pelo usuário, como nível crítico e níveis ideais de umidade do solo. Esses paramentos são variáveis conforme apresentado na Figura 7.

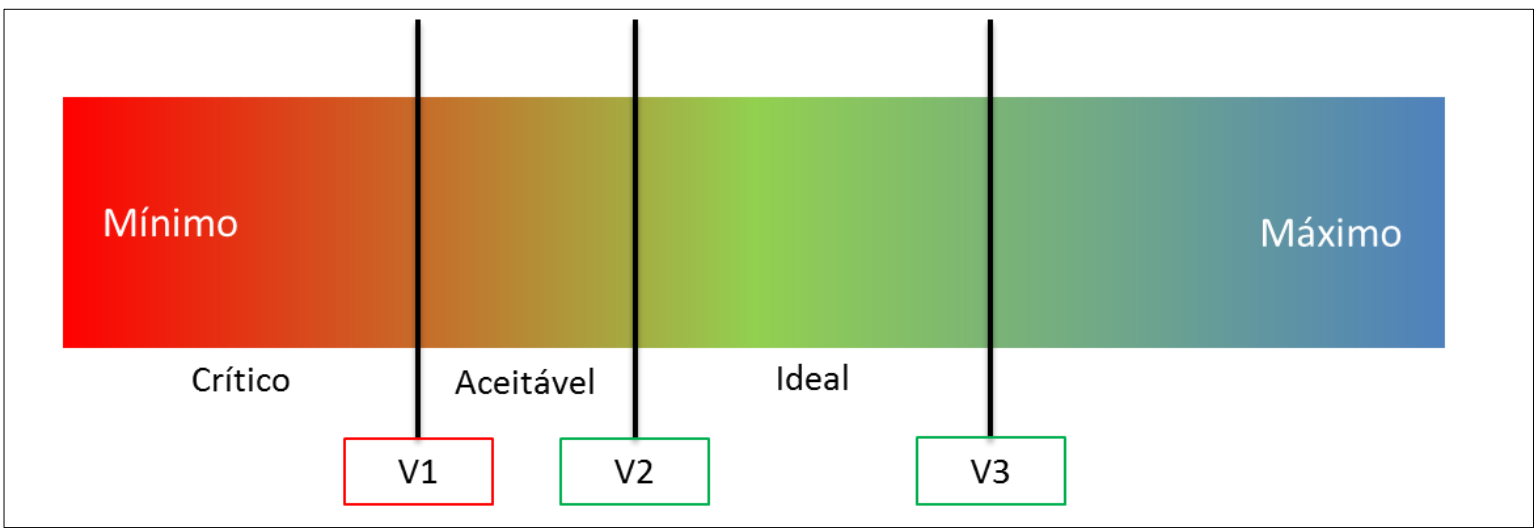

Figura 7 - Representação dos parâmetros de umidade.

Como se pode observar na Figura 7, V1 representa um valor crítico de umidade do solo e V2 e V3 valores ideais (mínimo e máximo respectivamente). O ideal é que os valores de umidade estejam entre $\boldsymbol{V} \mathbf{2}$ e $\boldsymbol{V} \mathbf{3}$, porém como o sistema busca também otimizar o consumo de água, levase em conta os fatores luminosidade e temperatura. Pois com a incidência forte de radiação de luz solar e a temperatura elevada, parte da agua será evaporada, o que representa desperdício. Uma forma encontrada de contornar essa situação é criando um terceiro intervalo de valores de umidade, entre $\mathbf{V} \mathbf{1}$ e $\boldsymbol{V} \mathbf{2}$, que dependendo dos fatores luminosidade e de temperatura, poderá ser considerado ideal.

Assim a umidade nunca poderá atingir o nível crítico. Já no nível aceitável a irrigação será ativada caso o nível de luminosidade e de temperatura estejam baixos e só será desligado ao atingir V3. No nível ideal a irrigação permanecerá desligada. Uma ilustração do algoritmo pode ser visualizada na Figura 8. 


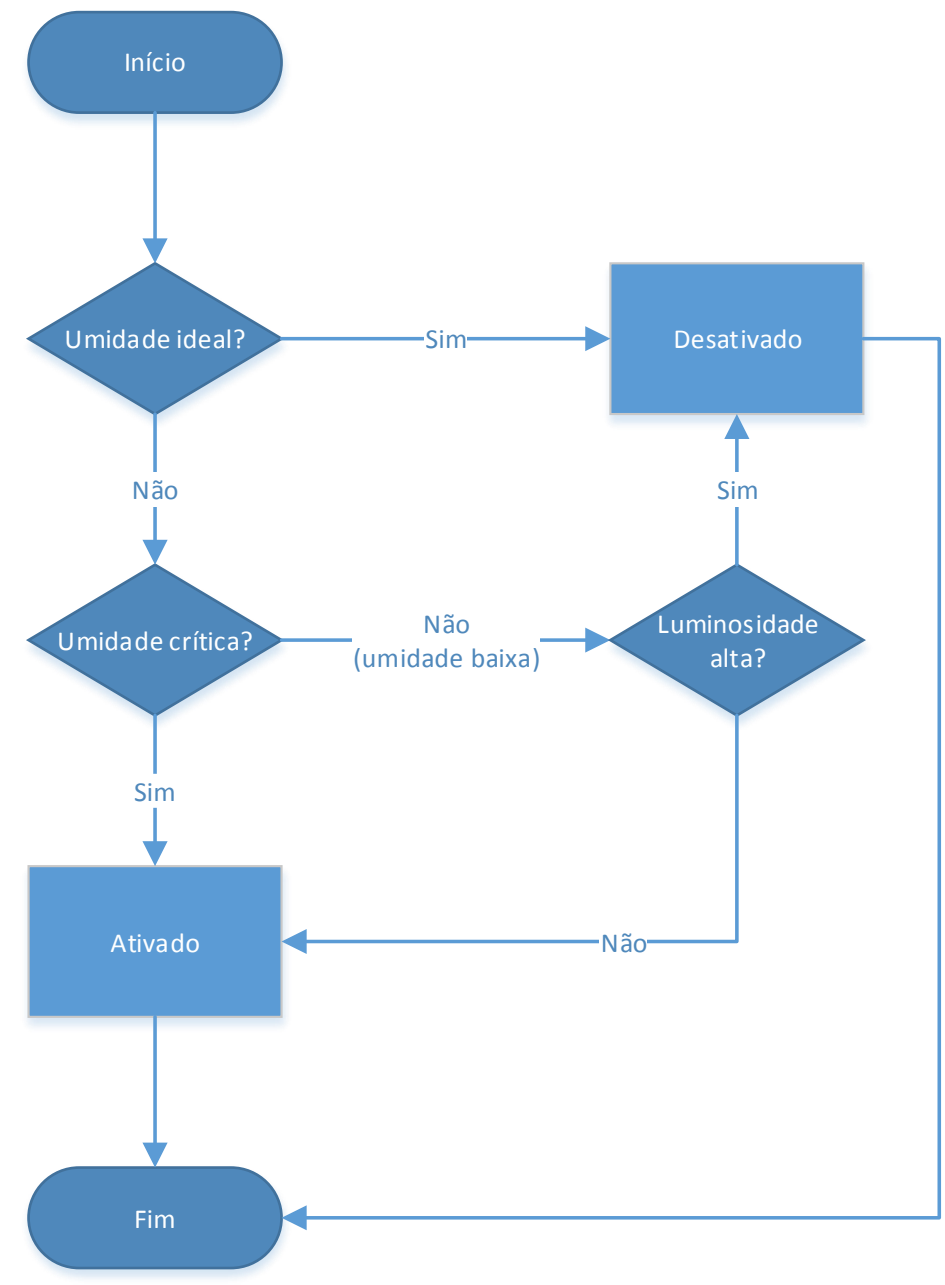

Figura 8 - Fluxograma do algoritmo utilizado.

\subsection{Segurança do Sistema e Controle de falhas}

Segundo (WEBER, 2003), todos componentes físicos envelhecem e sofrem com interferências externas, seja ambientais ou humanas. O software, e também os projetos de software e hardware, são vítimas de sua alta complexidade. Assim, todo sistema computacional é suscetível a falhas

No caso do Sistema de Irrigação, se os sensores falharem, causaria o excesso/escassez de volume de água, o que provocaria grandes prejuízos ao cultivo. Para contornar essas falhas, utilizou-se a redundância no uso dos sensores. Assim, foi adicionado mais um sensor de umidade e um de luminosidade. O sistema realiza um cálculo da diferença entre os valores dos sensores, e caso haja discrepância entre esses, o usuário será notificado. Além disso, é determinado um tempo limite para irrigação, e se esse tempo for atingido à mesma será desativada, e da mesma forma como a anterior o usuário será notificado pela anormalidade.

\section{SOFTWARE}

No desenvolvimento do Sistema de Software foram utilizados IDE, editores de textos e editores de imagens, para programação e modelagem de interface gráfica, as mesmas podem ser visualizadas na listagem a seguir: 
- Arduino IDE 1.0.4;

- NetBeans IDE 7.4;

- Fritzing;

- Adobe Dreamweaver.

\subsection{Arduino IDE}

O Arduino IDE é disponibilizado no site oficial do Arduino, descrito nas Referências Bibliográficas deste trabalho; antes de utiliza-la é necessário instalar o driver, para comunicação Serial da aplicação com as diferentes distribuições de sistemas embarcados do fabricante. $O$ mecanismo foi desenvolvido em Java, e permite ao usuário uma programação de alto nível, semelhante a Java.

Por meio do software, o Arduino foi programado para iniciar as interfaces digitais e analógicas utilizadas, criar interrupções a cada 3 segundos para ler os valores dos sensores, e sincronizar o tempo com o RTC DS1302. Além disso, foram incluídas algumas bibliotecas como a TimerOne.h para manipulação das interrupções, EEPROM.h para o serviço de agendamento e gravação de dados na memoria EEPROM do hardware, e as Wire.h e RTClib.h para uso do módulo de relógio RTC DS1302.

\subsection{Aplicação Java Sensor-Monitor}

A aplicação denominada Sensor-Monitor foi desenvolvida para um servidor, permitindo que o usuário mantenha-se informado sobre a situação da irrigação e que também possa interferir com maior controle sobre a mesma.

A aplicação foi desenvolvida através do NetBeans IDE, pela linguagem de programação Java. Essa linguagem foi escolhida, pois garante ao desenvolvedor e ao usuário como resultado um software que é portátil através de maquinas de arquiteturas múltiplas, sistemas operativos e interfaces de uso gráfico, seguras e de alto desempenho (GAVIDIA, 1997).

\subsection{Funcionamento da aplicação Sensor-Monitor em Desktop}

A aplicação Sensor-Monitor, como toda aplicação Java, é um diretório contendo vários recursos, o diretório da aplicação possui um tamanho aproximado de 10MB, seu arquivo executável é o SensorMonitor.jar, também é possível executar cada classe do projeto separadamente através do Prompt de Comando em sistema Windows ou pelo Terminal em sistema Linux.

Com todos os procedimentos de instalação realizados, é possível executar o software. A primeira ação ao executar o mesmo, é a chamada da Classe SAgenda, definida como a principal no desenvolvimento do software. Inicialmente a classe faz as importações de outras classes utilizadas em seu escopo, como as classes utilizadas para modelagem de tempo e de arquivo, as responsáveis pela comunicação Serial da aplicação, da biblioteca RXTXcomm e outras para construção de funções de utilidade geral do software.

Após as importações, é executado o método main da classe SAgenda, nele é iniciado o Construtor, que é responsável por iniciar a comunicação Serial e pela inicialização de duas Threads, 
para executarem simultaneamente, uma encarregada por interrupções quando dados estiverem disponíveis na Serial, e a outra para teste de conexão com o sistema.

O primeiro procedimento ao iniciar a comunicação Serial é a execução de uma função que lista as portas Seriais que estão sendo utilizadas, na interface gráfica o usuário seleciona a interface serial ao qual o Arduino do sistema de irrigação está conectada. A janela tanto em sistema Windows como em Linux podem ser visualizada na Figura 9.

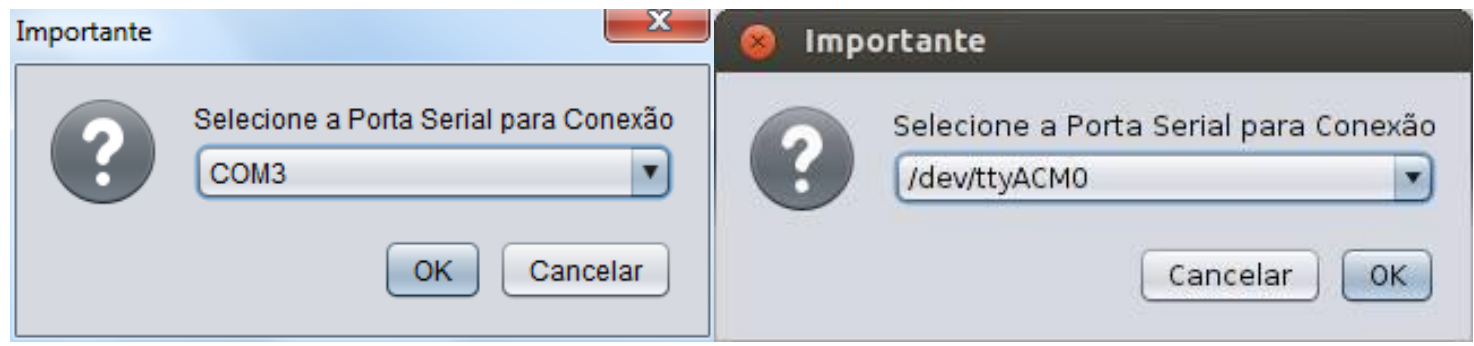

Figura 9 - Painel para escolha da Porta Serial em sistemas Windows e Linux.

Após a seleção da porta Serial, como foi visualizado na imagem anterior, o controle do sistema é inicializado e a interface gráfica do programa é exibida para o usuário com sucesso, como apresentado na Figura 10.

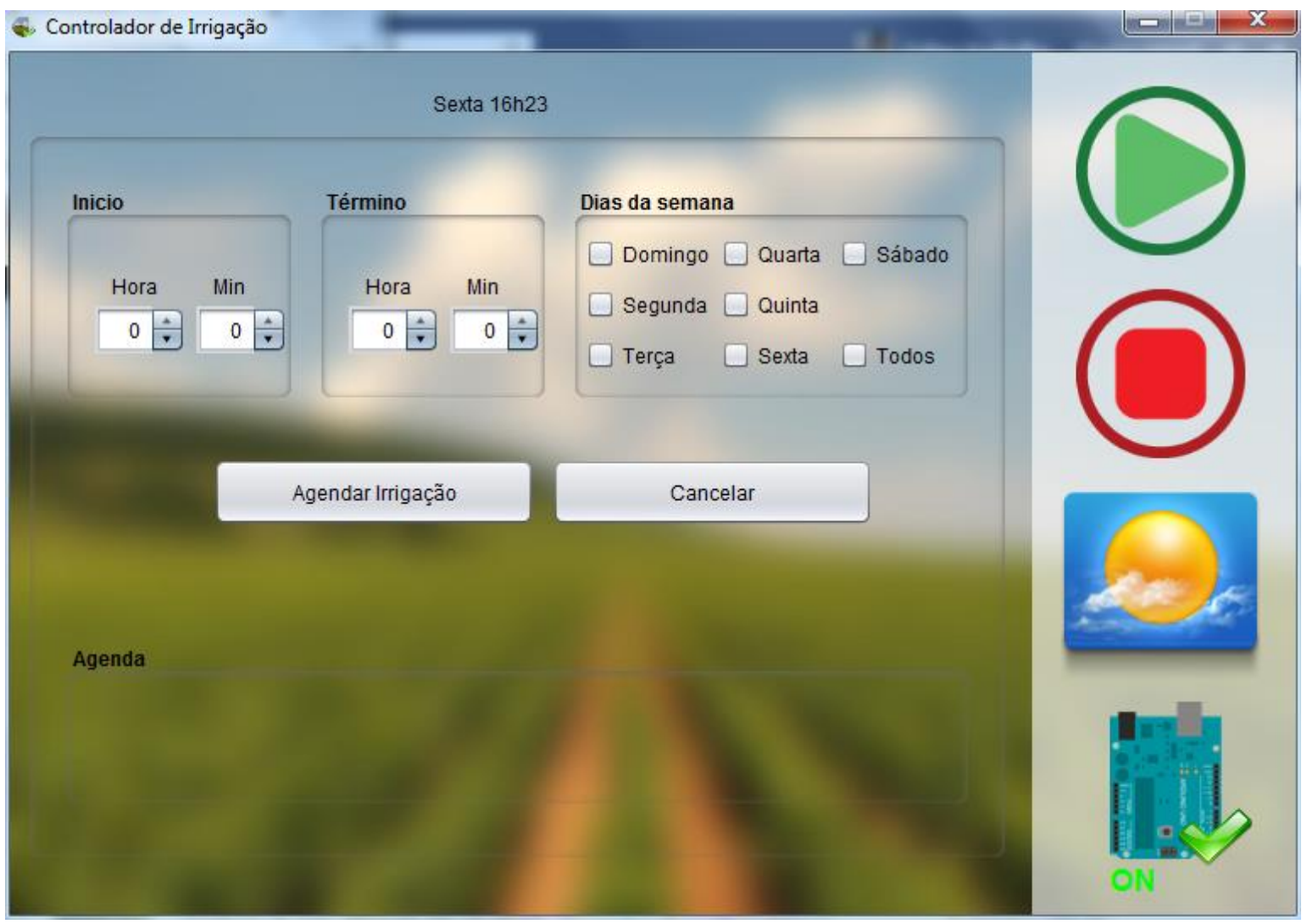

Figura 10 - Software Sensor-Monitor, Tela de Agenda.

A Figura do Arduino com o sinal de confirmação representa o status de conexão do mesmo com a aplicação. Se a porta serial não é a mesma do sistema de irrigação ou por outro motivo o sistema foi desconectado, será exibido para o usuário um status de problema de conexão, como ilustrado na Figura 11. 


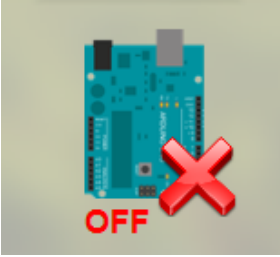

Figura 11 - Estado de Sistema de irrigação Desconectado.

$\mathrm{Na}$ interface gráfica da Agenda, ilustrado na Figura 10, o usuário terá o controle para estipular o horário inicial e o horário final da irrigação, e mais ao lado, a marcação dos dias da semana em que deve ser realizado a ação, após esse processo, o botão "Agendar Irrigação" irá confirmar a irrigação e enviar via Serial os dados para o Arduino, se obtiver sucesso a aplicação irá mostrar para o usuário uma mensagem de sucesso.

Após a mensagem de sucesso será exibido para o usuário no campo "Agenda", a nova Agenda de irrigação, um exemplo de uma irrigação marcada para iniciar às 06:20, e encerra às 08:40, por quatro vezes na semana, pode ser visualizado na Figura 12.

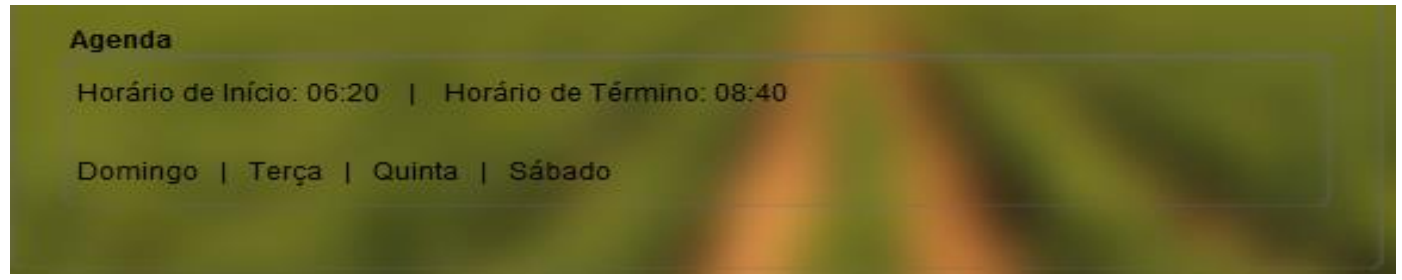

Figura 12 - Exemplo de agendamento de irrigação.

Já se o usuário marcou um horário inválido (horário final maior que horário inicial ou horários iguais) ou não marcou um dia da semana outra mensagem será exibida, como ilustrado na Figura 13.

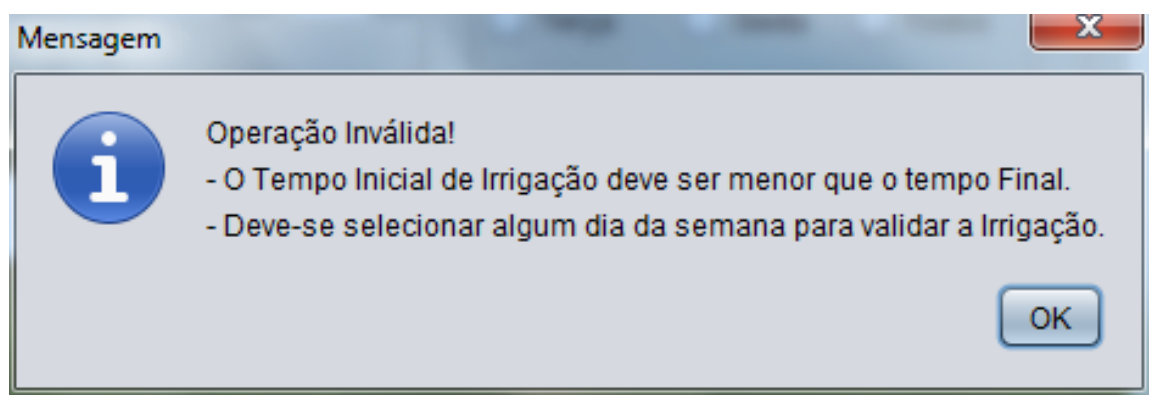

Figura 13 - Mensagem de Operação Inválida no agendamento de irrigação.

Outra possibilidade é o usuário tentar agendar o novo horário com o sistema desconectado, nesse caso ele receberá uma mensagem, informando que há falta de conexão.

Além de marcar uma Irrigação o usuário tem a opção de cancelar uma já existente, através do botão Cancelar, ilustrado na Figura 10, ao canelar a irrigação será enviado via Serial para o Arduino uma mensagem de "cancel", ao qualquer horário ou irrigação em curso estará desativada, após a ação o usuário receberá uma mensagem de confirmação, caso o sistema esteja desconectado será exibido a mensagem de operação invalida.

Ainda na tela de Agenda, está disponível para o usuário a ativação e desativação imediata da irrigação, sem a necessidade de agendamento, o botão play e o botão stop localizados no menu da tela executam as respectivas funções citadas. 
Após todas as opções na tela de Agenda, o usuário pode optar pelo monitoramento da irrigação, ao qual corresponde a outra tela, na Figura 10 ao clicar no botão de Monitoramento abaixo do botão de stop, é executada a classe SMonitor, essa classe é responsável por apresentar ao usuário as variáveis de temperatura, luminosidade e umidade, coletadas no campo, além de um gráfico com um dos valores, atualizando essas variáveis a cada três segundos, frequência em que o Arduino as atualiza. A Figura 14 demostra o monitoramento conectada ao Arduino em pleno funcionamento.

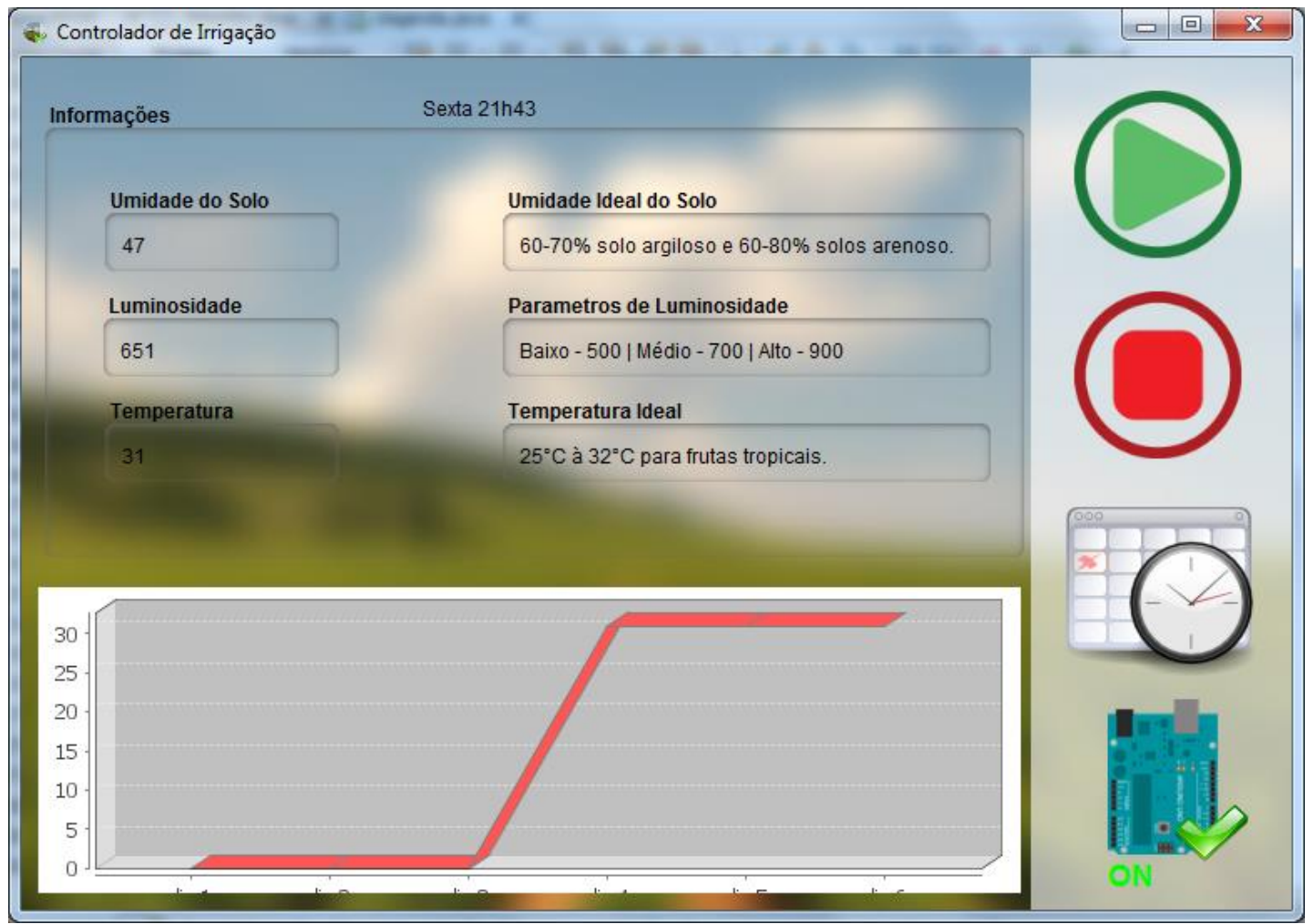

Figura 14 - Software no Monitoramento da Irrigação.

A tela de Monitoramento possui as mesmas opções do que a de Agenda, sendo o ícone de retornar a Agenda o diferencial.

\subsection{Acesso via internet}

O acesso do sistema através da internet é vital, levando em consideração a quantidade de aparelhos digitais hoje com capacidade de navegação através da internet e acesso a recursos multimídias oferecidos pelas grandes empresas online, seja uma Smart TV, um Tablet, Smartphone ou os próprios computadores pessoais.

Dessa forma fez-se necessário uma adaptação do software Sensor-Monitor para que os dados do sistema fossem repassados para um web-server, com armazenamento desses dados em um banco de dados. O serviço utilizado para conectar o servidor local ao web-server e tratar as requisições dos clientes foi o PHP. Assim o cliente teria acesso aos dados de temperatura, luminosidade, umidade e outras informações, além do controle de ativação por meio da Internet.

A interface de desenvolvimento utilizada foi o Adobe Dreamweaver para criação das páginas, que permite o teste em diferentes navegadores e plataformas, sendo possível desenvolver uma página de simples adaptação para qualquer dispositivo. A seguir podem-se 
visualizar as páginas, na Figura 15 em um browser de Computador Pessoal e na Figura 16 em um smartphone.

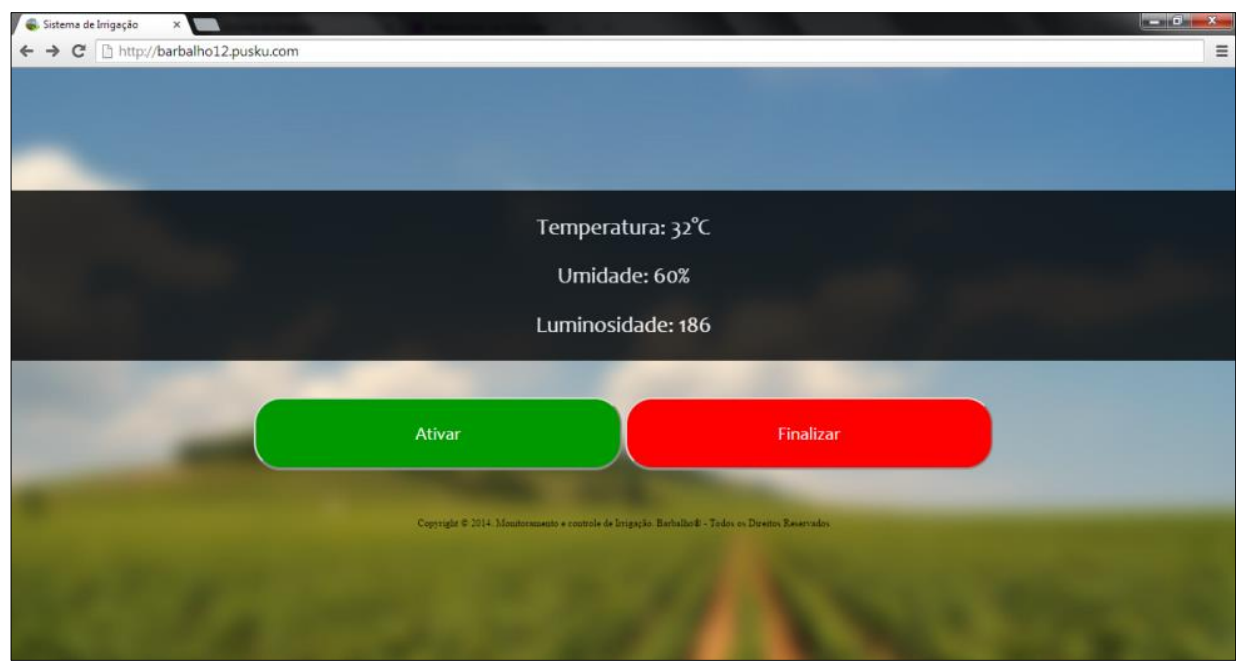

Figura 15 - Pagina Web acessada por meio de um Computador Pessoal.

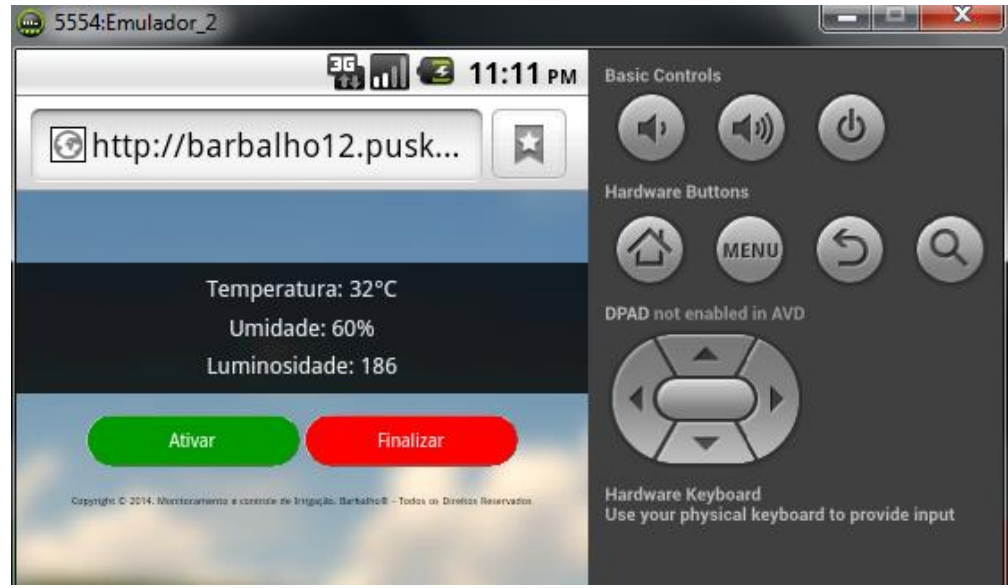

Figura 16 - Pagina Web acessada por meio de um simulador de Smartphone.

Como pode ser visualizado nas figuras anteriores, estão disponíveis para o usuário os dados captados pelos sensores e duas opções de iniciar e de finalizar a irrigação. Como boa prática de segurança, para ter acesso a página o usuário precisa fornecer um usuário e senha, inicialmente únicos.

\section{CONCLUSÃO}

Neste trabalho foi exposta uma alternativa automatizada para um sistema de irrigação, uma vez que atualmente grande parte desses sistemas ainda é controlado manualmente e isso implica em desperdício de recursos naturais, principalmente, a água.

O desenvolvimento do projeto, desde a coleta das informações até o produto final, que é a informação disponível, íntegra e autêntica para o usuário passa por diversas etapas. Adotando-se um sistema seguro, livre (aberto aos desenvolvedores), de baixo custo financeiro e simples.

Como trabalhos futuros pretende-se adicionar mais funcionalidades ao sistema, tais como aumentar o número de nós da rede, permitindo assim aplicar o sistema em irrigações de grande 
porte, prover mais segurança e estrutura aos serviços pela internet, aumentando assim a eficiência do sistema.

\section{REFERÊNCIAS BIBLIOGRÁFICAS}

1. AQUINO, J.F.S. Plataformas de desenvolvimento para dispositivos móveis. Rio De Janeiro, Dezembro de 2007.

2. ARDUINO. Disponível em:<http://www.arduino.cc/>. Acesso em: 06 de Junho de 2013.

3. CAVAlCANTE, M.A., TAVOlARO C.R.C., MOLISANI, E. Física com Arduino para iniciantes. Revista Brasileira de Ensino de Física, v. 33, n. 4, 4503 (2011).

4. DIMARZIO, JEROME F.; Android: A programmer's Guide, New York. McGrawHill E-Book, 2008.

5. GAVIDIA, J.J.V. Programação funcional usando Java. 1997. Dissertação (Mestrado) - Instituto de Informática, Universidade Federal do Rio Grande do Sul. Porto Alegre. Julho de 1997.

6. ISTO É DINHEIRO. Uso de smartphones dobrou no último ano. Disponível em: <http://www.istoedinheiro.com.br/noticias/117403_USO+DE+SMARTPHONES+DOBROU+NO +ULTIMO+ANO+APONTA+PESQUISA>. Acesso em: Agosto de 2013.

7. JÚNIOR, M.A.P., CASTRO, R.O. Estudo de caso da plataforma Android com Interfaces Adaptativas. LUMNI, v. 1, n. 1, (2011).

8. LIMA, C.B DE, VILLAÇA, M.V.M. AVR e Arduino técnicas de projetos. Florianópolis: Edição dos Autores, 2012.632p.

9. LISTA, A.M. Projeto didático de Pesquisa: Informática. São Paulo: DCL, 2007. 776p.

10. PINHEIRO, J.M.S. As Redes com ZigBee. 2004. Disponível em: <http://www.projetoderedes.com.br/artigos/artigozigbee.php> Acesso em: 13 Julho de 2013.

11. QUEIROZ, T.M DE, BOTERL, T.A., FRIZZONE, J.A. Desenvolvimento de software e hardware para irrigação de precisão usando pivô central. Engenharia Agrícola, Jaboticabal, v.28, n.1, p.44-54, jan./mar. 2008.

12. ROBOCORE. Disponível em:<http://www.robocore.net/>. Acesso em: 06 de Junho de 2013.

13. RXTX. Disponível em:<http://rxtx.qbang.org/wiki/index.php/>. Acesso em: 23 de Dezembro de 2013.

14. SILVA, I.L.S DA. Sistema de Irrigação Automatizado Baseado na Umidade do Solo. Instituto de Estudos Superiores da Amazônia (IESAM). Belém - PA. 2007.

15. SOUZA, A.R., PAIXÃO, A.C., UZÊDA, D.D., DIAS, M.A., Duarte, S., Amorim H.S. A placa Arduino: uma opção de baixo custo para experiências de física assistidas pelo PC. Revista Brasileira de Ensino de Física, v. 33, n. 1, 1702 (2011).

16. WEBER, T.S. Tolerância a falhas: conceitos e exemplos. Apostila do Programa de PósGraduação-Instituto de Informática-UFRGS. Porto Alegre. 2003. 